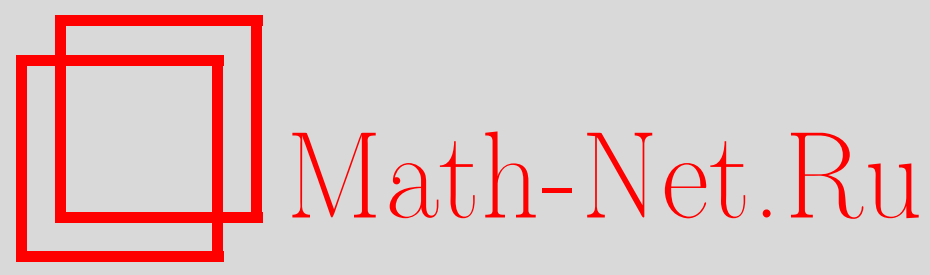

Ю. Д. Салманов, О гладкости обобщенного решения краевой задачи для некоторых вырождающихся нелинейных обыкновенных дифференциальных уравнений, Матем. заметки, 1998, том 63, выпуск 6, 882-890

DOI: https://doi.org/10.4213/mzm1359

Использование Общероссийского математического портала Math-Net.Ru подразумевает, что вы прочитали и согласны с пользовательским соглашением http://www.mathnet.ru/rus/agreement

Параметры загрузки:

IP: 54.205 .225 .156

26 апреля 2023 г., 14:27:13

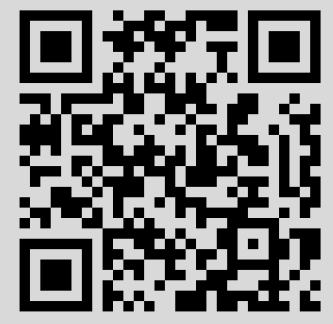




\section{О ГЛАДКОСТИ ОБОБШЕННОГО РЕШЕНИЯ КРАЕВОЙ ЗАДАЧИ ДЛЯ НЕКОТОРЫХ ВЫРОЖДАЮЩИХСЯ НЕЛИНЕЙНЫХ ОБЫКНОВЕННЫХ ДИФФЕРЕНЦИАЛЬНЫХ УРАВНЕНИЙ}

\section{ю. Д. Салманов}

В работе изучается вариационным методом краевая задача первого рода для некоторого класса нелинейных обыкновенных дифференциальных уравнений порядка $2 r$ с сильным вырождением на концах интервала $(a, b)$. Получено неравенство, в котором норма решения $U$ рассматриваемой задачи в смысле $W_{p, \alpha}^{r}(a, b)$ оценивается сверху нормами заданных функций $\Phi(x)$ и $F(x)$.

Библиография: 4 названия.

Изучим дифференщиальные свойства обобщенного решения вплоть до границы в зависимости от дифференциальных свойств коэффициентов уравнения (теорема 3 ). Установим неравенства типа неравенства коэрцитивности решения (см. формулы (42), (43)). Пусть $W_{p, \alpha}^{r}=W_{p, \alpha}^{r}(a, b)(\Delta=(a, b))$ - пространство функций $f(x)$, для которых имеет смысл конечная норма (см. $[1$, гл. 10]):

$$
\|f\|_{W_{p, \alpha}^{r}(\Delta)}=\|f\|_{p, \Delta}+\left\|\frac{f^{(r)}}{\rho^{\alpha}}\right\|_{p, \Delta}<+\infty
$$

где $r \geqslant 1$ - натуральное число, $\alpha, p(1<p<+\infty)$ - действительные числа,

$$
\begin{gathered}
\|\cdot\|_{p, \Delta}=\|\cdot\|_{L_{p}(\Delta)}, \quad f^{(k)}=f^{(k)}(x)=\frac{d^{k} f(x)}{d x^{k}}, \quad k \leqslant r, \\
\rho=\rho(x)=\min \{x-a, b-x\} \quad \forall x \in \Delta=(a, b) .
\end{gathered}
$$

Пусть

$$
0<r+\alpha-\frac{1}{p}<r
$$

и $s_{0}$ - натуральное число такое, что

$$
s_{0}-1<r+\alpha-\frac{1}{p} \leqslant s_{0}, \quad \frac{r}{2} \leqslant s_{0} \leqslant r .
$$

При условиях (1), (2) определим подпространство (см. [1, гл. 10])

$$
W_{0}=\stackrel{\circ}{W}_{p, \alpha}^{r}=\left\{f \in W_{p, \alpha}^{r}(a, b): f^{(k)}(a)=f^{(k)}(b)=0, k=0,1, \ldots, s_{0}-1\right\} .
$$


Зададим функцию $\Phi(x) \in W_{p, \alpha}^{r}(a, b)$ и обозначим через $W_{\Phi}$ множество $f(x) \in W_{p, \alpha}^{r}(a, b)$ таких, что $f(x)-\Phi(x) \in W_{0}$. При условиях (1), (2) справедливы неравенства (см. [2]-[4])

$$
\begin{aligned}
& \left\|\frac{f^{(k)}}{\rho^{\alpha+r-k}}\right\|_{p, \Delta} \leqslant c_{0}\left\|\frac{f^{(r)}}{\rho^{\alpha}}\right\|_{p, \Delta} \quad \forall f \in W_{0}, \quad k \leqslant r, \\
& \left\|\frac{f^{(k)}}{\rho^{\alpha+r-\beta_{k}}}\right\|_{p, \Delta} \leqslant c_{1}\|f\|_{W_{p, \alpha}^{r}(\Delta)} \quad \forall f \in W_{p, \alpha}^{r}(\Delta), \quad k \leqslant r, \\
& \beta_{k}=\left\{\begin{array}{ll}
k, & k \geqslant s_{0}, \\
s_{0}, & k \leqslant s_{0},
\end{array} \quad k \leqslant r .\right.
\end{aligned}
$$

Пусть каждому неотрицательному целому числу $k(k \leqslant r)$ поставлена в соответствие измеримая на $(a, b)$ функция $a_{k}(x)$ такая, что

$\frac{c_{2}}{\rho^{p_{k}\left(r+\alpha-\beta_{k}\right)}(x)} \leqslant a_{k}(x) \leqslant \frac{c_{3}}{\rho^{p_{k}\left(r+\alpha-\beta_{k}\right)}(x)}, \quad 2 \leqslant p_{k} \leqslant p, \quad k=0,1, \ldots, r-1, \quad p_{r}=p$,

где $c_{2}$ и $c_{3}$ - положительные постоянные, не зависящие от $x \in \Delta=(a, b)$. Введем в рассмотрение интеграл

$$
E(f)=\int_{a}^{b} \sum_{k \leqslant r} \frac{1}{p_{k}} a_{k}(x)\left|f^{(k)}(x)\right|^{p_{k}} d x,
$$

который, очевидно, конечен для $f \in W_{p, \alpha}^{r}(a, b)$ при условиях (1), (2), (5). Положим $H(f)=E(f)-(F, f)$, где $(F, f)$ - скалярное произведение функций $F(x)$ и $f(x)$ на $\Delta$ и функция $F(x)$ такая, что

$$
\left\|\rho^{\alpha+r-s_{0}} F\right\|_{q, \Delta}<+\infty, \quad \frac{1}{p}+\frac{1}{q}=1 .
$$

При условиях $(1),(2),(5),(6)$ функционал $H(f)$ ограничен в классе $W_{\Phi}$ снизу. Положим

$$
\inf _{f \in W_{\Phi}} H(f)=\lambda .
$$

Теорема 1. При условиях (1), (2), (5), (6) существует и притом единственная функиия $U(x) \in W_{\Phi}$ такая, что

$$
\min _{f \in W_{\Phi}} H(f)=H(U)=\lambda,
$$

удовлетворяющая равенству

$$
\int_{a}^{b}\left(\sum_{k \leqslant r} a_{k}(x)\left|U^{(k)}(x)\right|^{p_{k}-2} U^{(k)}(x) v^{(k)}(x)-F(x) v(x)\right) d x=0 \quad \forall f \in W_{0} .
$$

Функцию $U \in W_{\Phi}$, удовлетворяющую (8) при любой $v \in W_{0}$, будем назьвать обобщенным решением краевой задачи

$$
L U=F(x), \quad x \in(a, b), \quad U \in W_{\Phi},
$$

где

$$
L U=\sum_{k \leqslant r}(-1)^{k}\left(a_{k}(x)\left|U^{(k)}(x)\right|^{p_{k}-2} U^{(k)}(x)\right)^{(k)} .
$$


Теорема 2. Для решения $U \in W_{\Phi}$ вариационной задачи (7) имеет место неравенство

$$
\|U\|_{W_{p, \alpha}^{r}(\Delta)}^{p} \leqslant c_{4}\left(\|\Phi\|_{W_{p, \alpha}^{r}(\Delta)}^{\gamma_{0}}+\left\|\rho^{\alpha+r-s_{0}} F\right\|_{q, \Delta}^{q}\right)
$$

$2 \partial e$

$$
\gamma_{0}= \begin{cases}p, & \|\Phi\|_{W_{p, \alpha}^{r}(\Delta)} \geqslant 1 \\ \min _{k} p_{k}, & \|\Phi\|_{W_{p, \alpha}^{r}(\Delta)} \leqslant 1\end{cases}
$$

$c_{4}$ - постоянная, не зависящая от $\Phi$ и $F$.

Неравенство (9) устанавливается на основании тождества (8).

ТЕорема 3. Пусть выполняются условия (1), (2), (6), функиии $a_{k}(x)$, әде $k \leqslant r / 2$, $k=r$, имеют на интервале $(a, b)$ непрерывные производные до порядка $k$ включительно такие, что

$\left|a_{k}^{(s)}(x)\right| \leqslant \frac{c_{5}}{\rho^{p_{k}\left(r+\alpha-\beta_{k}\right)+s}(x)}, \quad x \in(a, b), \quad s \leqslant k, \quad 2 \leqslant p_{k} \leqslant p, \quad k \leqslant \frac{r}{2}, \quad p_{r}=p$,

а функиия $U \in W_{\Phi}$ удовлетворяет равенству $\left(a(x) \equiv a_{r}(x)\right)$

$$
\begin{aligned}
& \int_{a}^{b}\left(a(x)\left|U^{(r)}(x)\right|^{p-2} U^{(r)}(x) v^{(r)}(x)\right. \\
& \left.\quad+\sum_{k \leqslant r / 2} a_{k}(x)\left|U^{(k)}(x)\right|^{p_{k}-2} U^{(k)}(x) v^{(k)}(x)-F(x) v(x)\right) d x=0
\end{aligned}
$$

для всех $v \in W_{0}$. Тогда для функиии $\psi(x) \equiv a(x)\left|U^{(r)}(x)\right|^{p-2} U^{(r)}(x)$ имеют место следующие неравенства:

$$
\begin{gathered}
\left\|\rho^{\alpha} \psi\right\|_{q, \Delta} \leqslant c_{6}\|U\|_{W_{p, \alpha}^{r}(\Delta)}^{p-1}, \\
\left\|\rho^{\alpha+r+\left(l_{0}-q\right) /\left(q l_{0}\right)} \psi^{(r)}\right\|_{q, \Delta} \leqslant c_{7}\left(\|U\|_{W_{p, \alpha}^{r}(\Delta)}^{\lambda_{0}}+\left\|\rho^{\alpha+r-s_{0}} F\right\|_{q, \Delta}\right),
\end{gathered}
$$

əde

$$
\lambda_{0}=\left\{\begin{array}{ll}
p-1, & \|U\|_{W_{p, \alpha}^{r}(\Delta)} \geqslant 1, \\
\min _{k} p_{k}-1, & \|U\|_{W_{p, \alpha}^{r}(\Delta)} \leqslant 1,
\end{array} \quad l_{0}=\max _{k} q_{k}, \quad \frac{1}{p_{k}}+\frac{1}{q_{k}}=1,\right.
$$

$c_{6}, c_{7}$ - постоянные, не зависящие от $U$ и от $F, U$ соответственно.

ДокАЗАТЕЛЬСТво. Пусть $\sigma, \sigma^{\prime}, \sigma^{\prime \prime} \in(a, b)$ - интервалы с центрами в $x_{0} \in(a, b)$, радиусы которых соответственно равны $4^{-1} \rho\left(x_{0}\right), 2^{-1} \rho\left(x_{0}\right), \rho\left(x_{0}\right)$, где $\rho\left(x_{0}\right)=\min \left\{x_{0}-a\right.$, $\left.b-x_{0}\right\}$. Для любой функции $v(x) \in W_{0}$, финитной в $\sigma^{\prime}$, будем писать $v(x) \in W_{0}\left(\sigma^{\prime}\right)$. На основании $(11)$ имеем $(h \neq 0)$

$$
\begin{aligned}
& \int_{\sigma^{\prime}}\left(\frac{\Delta^{s}}{h^{s}}\left(a(x)\left|U^{(r)}(x)\right|^{p-2} U^{(r)}(x)\right) v^{(r)}(x)\right. \\
& +\sum_{k \leqslant r / 2} \frac{\Delta^{s}}{h^{s}}\left(a_{k}(x)\left|U^{(k)}(x)\right|^{p_{k}-2} U^{(k)}(x)\right) v^{(k)}(x) \\
& \left.-\frac{\Delta^{s} F(x)}{h^{s}} v(x)\right) d x=0 \quad \forall v \in W_{0}\left(\sigma^{\prime}\right),
\end{aligned}
$$


где $\Delta^{s} f(x)=\Delta_{h}^{s} f(x)$ есть $s$-кратная разность от функции $f(x)$ в точке $x$ с шагом $h$.

Введем в рассмотрение функцию $\eta(x) \in C^{(r+1)}(\mathbb{R})$, финитную в $\sigma^{\prime}$, с носителем $\Lambda_{\eta} \subset \sigma^{\prime}$, обладающую свойствами:

1) $\eta(x)=1$ на $\sigma$

2) $0 \leqslant \eta(x) \leqslant 1, x \in \mathbb{R}$;

3) $\left|\eta^{(k)}(x)\right|^{2} \leqslant c \eta(x), k \leqslant r+1$.

Положим (для достаточно малого $h \neq 0$ )

$$
A_{s}(x)=\frac{1}{\Gamma(r-1)} \int_{x_{0}}^{x}(x-t)^{r-1} \eta(t)\left|\frac{\Delta^{s} \psi(t)}{h^{s}}\right|^{q-1} \operatorname{sign} \frac{\Delta^{s} \psi(t)}{h^{s}} d t, \quad x \in \sigma^{\prime} .
$$

Имеем

$$
\left\|A_{s}\right\|_{p, \sigma^{\prime}} \ll|h|^{-s(q-1)}\left\|\Delta^{s} \psi\right\|_{q, \Lambda_{\eta}}^{q / p}
$$

Далее,

$$
\begin{aligned}
\left\|\Delta^{s} \psi\right\|_{q, \Lambda_{\eta}} & \ll \sum_{j=0}^{s}\|\psi(x+h j)\|_{q, \Lambda_{\eta}} \ll \rho^{-\alpha}\left(x_{0}\right) \sum_{j=0}^{s}\left(\int_{\Lambda_{\eta}}\left|\frac{U^{(r)}(x+h j)}{\rho^{\alpha}(x+h j)}\right|^{p} d x\right)^{1 / q} \\
& \ll \rho^{-\alpha}\left(x_{0}\right)\|U\|_{W_{p, \alpha}^{r}\left(\sigma^{\prime}\right)}^{p / q}
\end{aligned}
$$

Второе неравенство в (17) следует из неравенства (10) при $k=r, s=0\left(\beta_{r}=r\right)$ и того, что $\rho(x) \sim \rho\left(x_{0}\right)$ для любого $x \in \sigma^{\prime}$ (см. [2, с. 101]), последнее очевидно.

Из (16), (17) имеем

$$
\left\|A_{s}\right\|_{p, \sigma^{\prime}} \ll|h|^{-s(q-1)} \rho^{-\alpha q / p}\left(x_{0}\right)\|U\|_{W_{p, \alpha}^{r}\left(\sigma^{\prime}\right)} .
$$

Аналогично $(k \leqslant r)$,

$$
\left\|A_{s}^{(k)}\right\|_{p, \sigma^{\prime}} \ll|h|^{-s(q-1)} \rho^{-\alpha q / p}\left(x_{0}\right)\|U\|_{W_{p, \alpha}^{r}\left(\sigma^{\prime}\right)}
$$

Следовательно, функция $A_{s}(x)$ при фиксированном $h(h \neq 0)$ принадлежит классу $W_{0}\left(\sigma^{\prime}\right)$, поэтому в равенстве $(14)$ можно положить $v(x)=\eta(x) A_{s}(x)$. Тогда получим

$$
X\left(\sigma^{\prime}, s\right) \equiv \int_{\sigma^{\prime}} \eta^{2}(x)\left|\frac{\Delta^{s} \psi}{h^{s}}\right|^{q} d x=J_{1}\left(\sigma^{\prime}, s\right)+J_{2}\left(\sigma^{\prime}, s\right)+J_{3}\left(\sigma^{\prime}, s\right)
$$

где

$$
\begin{aligned}
& J_{1}\left(\sigma^{\prime}, s\right)=-\sum_{j=0}^{r-1} C_{r}^{j} \int_{\sigma^{\prime}} \frac{\Delta^{s} \psi(x)}{h^{s}} A_{s}^{(j)}(x) \eta^{(r-j)}(x) d x \\
& J_{2}\left(\sigma^{\prime}, s\right)=-\sum_{k \leqslant r / 2} \int_{\sigma^{\prime}} \frac{\Delta^{s}}{h^{s}}\left(a_{k}(x)\left|U^{(k)}(x)\right|^{p_{k}-2} U^{(k)}(x)\right)\left(A_{s}(x) \eta(x)\right)^{(k)} d x, \\
& J_{3}\left(\sigma^{\prime}, s\right)=\int_{\sigma^{\prime}} \frac{\Delta^{s} F(x)}{h^{s}} A_{s}(x) \eta(x) d x .
\end{aligned}
$$


Легко установить неравенства $(j \leqslant r, \tau \leqslant r+1)$

$$
\begin{gathered}
\left\|A_{s}^{(j)} \eta^{(\tau)}\right\|_{p_{k}, \sigma^{\prime}} \ll X^{1 / p}\left(\sigma^{\prime}, s\right), \\
\|\psi\|_{q, \sigma^{\prime}} \ll \rho^{-\alpha}\left(x_{0}\right)\|U\|_{W_{p, \alpha}^{r}\left(\sigma^{\prime}\right)}^{p / q}
\end{gathered}
$$

Теперь оценим интегралы $J_{1}\left(\sigma^{\prime}, 1\right), J_{2}\left(\sigma^{\prime}, 1\right), J_{3}\left(\sigma^{\prime}, 1\right)(s=1)$. Для интегралов, составляющих $J_{1}\left(\sigma^{\prime}, 1\right)$, имеем

$$
\begin{gathered}
\left|\int_{\sigma^{\prime}} \frac{\Delta \psi(x)}{h} A_{1}^{(j)}(x) \eta^{(r-j)}(x) d x\right|=\left|\int_{\sigma^{\prime}} \psi(x) \frac{\Delta_{-h}}{h}\left(A_{1}^{(j)}(x) \eta^{(r-j)}(x)\right) d x\right| \\
\ll\|\psi\|_{q, \sigma^{\prime}}\left\|\left(A_{1}^{(j)} \eta^{(r-j)}\right)^{\prime}\right\|_{p, \sigma^{\prime}} \ll \rho^{-\alpha}\left(x_{0}\right)\|U\|_{W_{p, \alpha}^{r}\left(\sigma^{\prime}\right)}^{p-1} X^{1 / p}\left(\sigma^{\prime}, 1\right) .
\end{gathered}
$$

Равенство в (21) написано в силу финитности функции $\eta(x)$, первое неравенство верно в силу неравенства Гёльдера и неравенства (5) из [2, с. 115] (в дальнейшем мы часто пользуемся этим неравенством, не упоминая об этом каждый раз), последнее следует из $(19),(20)$. Для интегралов, составляющих $J_{2}\left(\sigma^{\prime}, 1\right)$, имеем $(k+1 \leqslant r / 2+1 \leqslant r)$

$$
\begin{aligned}
& \left|\int_{\sigma^{\prime}} \frac{\Delta}{h}\left(a_{k}(x)\left|U^{(k)}(x)\right|^{p_{k}-2} U^{(k)}(x)\right)\left(A_{1} \eta\right)^{(k)} d x\right| \\
& \quad \leqslant\left\|a_{k}\left|U^{(k)}\right|^{p_{k}-1}\right\|_{q_{k}, \sigma^{\prime}}\left\|\left(A_{1} \eta\right)^{(k+1)}\right\|_{p_{k}, \sigma^{\prime}} \ll \rho^{-r-\alpha+\beta_{k}}\left(x_{0}\right)\|U\|_{W_{p_{k}, \alpha}^{r}\left(\sigma^{\prime}\right)} X^{1 / p}\left(\sigma^{\prime}, 1\right) .
\end{aligned}
$$

Для $J_{3}\left(\sigma^{\prime}, 1\right)$ имеем

$$
\begin{aligned}
\left|J_{3}\left(\sigma^{\prime}, 1\right)\right| & =\left|\int_{\sigma^{\prime}} F \frac{\Delta_{-h}}{h}\left(A_{1} \eta\right) d x\right| \leqslant\|F\|_{q, \sigma^{\prime}}\left\|\left(A_{1} \eta\right)^{\prime}\right\|_{p, \sigma^{\prime}} \\
& \ll \rho^{-r-\alpha+s_{0}}\left(x_{0}\right)\left\|\rho^{r+\alpha-s_{0}} F\right\|_{q, \sigma^{\prime}} X^{1 / p}\left(\sigma^{\prime}, 1\right) .
\end{aligned}
$$

В силу (18) (при $s=1)$ и (21)-(23) получаем

$$
X^{1 / q}\left(\sigma^{\prime}, 1\right) \ll \rho^{-r-\alpha}\left(x_{0}\right)\left(\|U\|_{W_{p, \alpha}^{r}\left(\sigma^{\prime}\right)}^{p-1}+\sum_{k \leqslant r / 2}\|U\|_{W_{p_{k}, \alpha}^{r}\left(\sigma^{\prime}\right)}^{p_{k}-1}+\left\|\rho^{r+\alpha-s_{0}} F\right\|_{q, \sigma^{\prime}}\right) \equiv S\left(\sigma^{\prime}\right) .
$$

Отсюда, так как $\eta(x)=1$ на $\sigma$, имеем

$$
\left\|\frac{\Delta \psi}{h}\right\|_{q, \sigma} \ll S\left(\sigma^{\prime}\right) .
$$

Заменим в последнем неравенстве $\sigma$ на $\sigma_{1}$ :

$$
\left\|\frac{\Delta \psi}{h}\right\|_{q, \sigma_{1}} \ll S\left(\sigma^{\prime}\right) .
$$

Пусть $\sigma \subset(a, b)$ - произвольно фиксированная окрестность точки $x_{0} \in(a, b)$ такая, что $\sigma \subset \sigma_{1} \subset \sigma^{\prime}$. Докажем, что

$$
\left\|\frac{\Delta^{r} \psi}{h^{r}}\right\|_{q, \sigma} \ll S\left(\sigma^{\prime}\right) .
$$


С этой целью введем в рассмотрение систему концентрических интервалов $\sigma_{r}, \sigma_{r}^{\prime}, \ldots$, $\sigma_{1}, \sigma_{1}^{\prime}$ с центрами в точке $x_{0} \in(a, b)$, для которых имеют место строгие вложения

$$
\sigma \equiv \sigma_{r} \subset \sigma_{r}^{\prime} \subset \cdots \subset \sigma_{1} \subset \sigma_{1}^{\prime} \equiv \sigma^{\prime} .
$$

Предположим, что установлены неравенства

$$
\left\|\frac{\Delta^{l} \psi}{h^{l}}\right\|_{q, \sigma_{l}} \ll S\left(\sigma^{\prime}\right), \quad l=1, \ldots, m,
$$

где $m$ - натуральное число такое, что $1 \leqslant m<r$. Покажем, что при этих условиях верно и неравенство

$$
\left\|\frac{\Delta^{m+1} \psi}{h^{m+1}}\right\|_{q, \sigma_{m+1}} \ll S\left(\sigma^{\prime}\right)
$$

и, следовательно, неравенство (26). Неравенство (28) при $l=1$ доказано (см. (25)).

Для каждого $l(l=1, \ldots, r)$ введем в рассмотрение функцию $\eta_{l}(x) \in C^{(r+1)}(\mathbb{R})$, финитную в $\sigma_{l}^{\prime}$, с носителем $\Lambda_{\eta_{l}}$, обладающую свойствами:

1) $\eta_{l}(x)=1$ на $\sigma_{l}$;

2) $0 \leqslant \eta_{l}(x) \leqslant 1, x \in \mathbb{R}$;

3) $\left|\eta^{(k)}(x)\right|^{2} \leqslant c \eta_{l}(x), k \leqslant r+1$, где $c$ - постоянная, не зависящая от точки $x \in \mathbb{R}$.

В формуле (15) $\eta(x), \sigma, \sigma^{\prime}$ заменим на $\eta_{l}(x), \sigma_{l}, \sigma_{l}^{\prime}$ соответственно.

Теперь в равенстве (18) положим $s=l=m+1$ и оценим интегралы $J_{i}\left(\sigma_{l}^{\prime}, l\right), i=1,2,3$. В силу (19) и $(27),(28)$ для интегралов, составляющих $J_{1}\left(\sigma_{l}^{\prime}, l\right)$, имеем

$$
\begin{aligned}
\left|\int_{\sigma_{l}^{\prime}} \frac{\Delta^{l} \psi}{h^{l}} A_{l}^{(j)} \eta^{(r-j)} d x\right| & =\left|\int_{\sigma_{l}^{\prime}} \frac{\Delta^{l-1} \psi}{h^{l-1}} \frac{\Delta_{-h}}{h}\left(A_{l}^{(j)} \eta^{(r-j)}\right) d x\right| \\
& \leqslant\left\|\frac{\Delta^{l-1} \psi}{h^{l-1}}\right\|_{q, \Lambda_{\eta_{l}}}\left\|\left(A_{l}^{(j)} \eta_{l}^{(r-j)}\right)^{\prime}\right\|_{p, \sigma^{\prime}} \\
& \ll\left\|\frac{\Delta^{l-1} \psi}{h^{l-1}}\right\|_{q, \sigma_{l-1}} X^{1 / p}\left(\sigma_{l}^{\prime}, l\right) \ll S\left(\sigma^{\prime}\right) X^{1 / p}\left(\sigma_{l}^{\prime}, l\right) .
\end{aligned}
$$

Оценим интегралы, составляющие $J_{2}\left(\sigma_{l}^{\prime}, l\right)$. Рассмотрим сначала случай $l \leqslant k$ :

$$
\begin{aligned}
E & \equiv\left|\int_{\sigma_{l}^{\prime}} \frac{\Delta^{l}}{h^{l}}\left(a_{k}(x)\left|U^{(k)}(x)\right|^{p_{k}-2} U^{(k)}(x)\right)\left(A_{l} \eta_{l}\right)^{(k)} d x\right| \\
& \ll\left\|a_{k}\left|U^{(k)}\right|^{p_{k}-1}\right\|_{q_{k}, \sigma_{l}^{\prime}}\left\|\frac{\Delta_{-h}^{l}}{h^{l}}\left(A_{l} \eta_{l}\right)^{(k)}\right\|_{p_{k}, \sigma_{l}^{\prime}} \\
& \ll \rho^{-r-\alpha+\beta_{k}}\left(x_{0}\right)\left\|\frac{U^{(k)}}{\rho^{r+\alpha-\beta_{k}}}\right\|_{p_{k}, \sigma_{l}^{\prime}}^{p_{k}-1}\left\|\left(A_{l} \eta_{l}\right)^{(k+l)}\right\|_{p_{k}, \sigma_{l}^{\prime}} \\
& \ll \rho^{-r-\alpha+\beta_{k}}\left(x_{0}\right)\|U\|_{W_{p_{k}, \alpha}^{r}\left(\sigma_{l}^{\prime}\right)}^{p_{k}-1} X^{1 / p}\left(\sigma_{l}^{\prime}, l\right) .
\end{aligned}
$$

Равенство в цепи соотношений (31) верно в силу финитности функции $\eta_{l}(x)$, первое неравенство очевидно, второе написано на основании неравенства (10) и того факта, что 
$\rho(x) \sim \rho\left(x_{0}\right)$ для любого $x \in \sigma_{l}^{\prime}[2$, с. 101], последнее следует из (3) и (19). Пусть $k<l \leqslant r$. Тогда

$$
\begin{aligned}
E & \ll\left\|\left(a_{k}\left|U^{(k)}\right|^{p_{k}-2} U^{(k)}\right)^{(k)}\right\|_{q_{k}, \sigma_{l}^{\prime}}\left\|\left(A_{l} \eta_{l}\right)^{(l)}\right\|_{p_{k}, \sigma_{l}^{\prime}} \\
& \ll \sum_{n=0}^{k} \rho^{-p_{k}\left(r+\alpha-\beta_{k}\right)-k+n}\left(x_{0}\right)\left\|\left(\left|U^{(k)}\right|^{p_{k}-2} U^{(k)}\right)^{(n)}\right\|_{q_{k}, \sigma_{l}^{\prime}} X^{1 / p}\left(\sigma_{l}^{\prime}, l\right) .
\end{aligned}
$$

Последнее неравенство в цепи (32) следует из неравенств (10), (19), а также неравенств (1) из [2, с. 101].

Теперь получим оценку для интеграла

$$
\left\|E_{n}\right\| \equiv\left\|\left(\left|U^{(k)}\right|^{p_{k}-2} U^{(k)}\right)^{(n)}\right\|_{q_{k}, \sigma_{l}^{\prime}} .
$$

С этой целью оценим $L_{q_{k}}\left(\sigma_{l}^{\prime}\right)$-норму каждого слагаемого из следуюших равенств:

$$
\begin{aligned}
E_{2 j}= & \left|U^{(k)}(x)\right|^{p_{k}-2(j+1)}\left(\sum_{s=0}^{2 j-1} c_{k s} U^{(k+2 j-s)}(x)\left(U^{(k+1)}(x)\right)^{s}\left(U^{(k)}(x)\right)^{2 j-s}\right. \\
& \left.+\sum_{s=1}^{j-1} d_{k s} U^{(k+2(j-s))}(x)\left(U^{(k+2)}(x)\right)^{s}\left(U^{(k)}(x)\right)^{2 j-s}\right), \quad 2 \leqslant 2 j \leqslant k, \quad(33) \\
E_{2 j-1}= & \left|U^{(k)}(x)\right|^{p_{k}-2 j}\left(\sum_{s=0}^{2(j-1)} c_{k s}^{\prime} U^{(k+2 j-1-s)}(x)\left(U^{(k+1)}(x)\right)^{s}\left(U^{(k)}(x)\right)^{2(j-1)-s}\right. \\
& \left.+\sum_{s=1}^{j-1} d_{k s}^{\prime} U^{(k+2(j-s)-1)}(x)\left(U^{(k+2)}(x)\right)^{s}\left(U^{(k)}(x)\right)^{2(j-1)-s}\right), \quad 1 \leqslant 2 j-1 \leqslant k,
\end{aligned}
$$

где $c_{k s}, d_{k s}, c_{k s}^{\prime}, d_{k s}^{\prime}-$ константы, точные значения которых для нас несущественны. Воспользуемся неравенствами (1) из [2, с. 101] для оценки слагаемых первой суммы из (33):

$$
\begin{aligned}
& \left\|\left|U^{(k)}\right|^{p_{k}-2-s}\left(U^{(k+1)}\right)^{s} U^{(k+2 j-s)}\right\|_{q_{k}, \sigma_{l}^{\prime}} \\
& \quad \ll \rho^{\nu_{1}}\left(x_{0}\right)\left\|\left|\frac{U^{(k)}}{\rho^{r+\alpha-\beta_{k}}}\right|^{p_{k}-s-2}\left|\frac{U^{(k+1)}}{\rho^{r+\alpha-\beta_{k+1}}}\right|^{s}\left|\frac{U^{(k+2 j-s)}}{\rho^{r+\alpha-\beta_{k+2 j-s}}}\right|\right\|_{q_{k}, \sigma_{l}^{\prime}} \equiv \rho^{\nu_{1}}\left(x_{0}\right) Q,
\end{aligned}
$$

где $\nu_{1}=\left(p_{k}-2-s\right)\left(r+\alpha-\beta_{k}\right)+s\left(r+\alpha-\beta_{k+1}\right)+r+\alpha-\beta_{k+2 j-s}$. Пусть $s=0$, $p_{k}>2$. Применяя неравенство Гёльдера с показателями

$$
\lambda_{1}=\frac{p_{k}}{q_{k}\left(p_{k}-2\right)}, \quad \lambda_{2}=\frac{p_{k}}{q_{k}}
$$

получим

$$
Q \leqslant\left\|\frac{U^{(k)}}{\rho^{r+\alpha-\beta_{k}}}\right\|_{p_{k}, \sigma_{l}^{\prime}}^{p_{k}-2}\left\|\frac{U^{(k+2 j)}}{\rho^{r+\alpha-\beta_{k+2 j} j}}\right\|_{p_{k}, \sigma_{l}^{\prime}} \ll\|U\|_{W_{p_{k}, \alpha}^{r}\left(\sigma_{l}^{\prime}\right)}^{p_{k}-1} .
$$


Последнее неравенство в (36) следует из (3). Если $p_{k}=2$, то $q_{k}=2$. Поэтому

$$
Q=\left\|\frac{U^{(k+2 j)}}{\rho^{r+\alpha-\beta_{k+2 j}}}\right\|_{2, \sigma_{l}^{\prime}} \ll\|U\|_{W_{2, \alpha}^{r}\left(\sigma_{l}^{\prime}\right)}
$$

Пусть $s \geqslant 1$. Если $p_{k}-2-s=0$, то $p_{k}-2 \geqslant 1$. Поэтому

$$
Q \leqslant\left\|\frac{U^{(k+1)}}{\rho^{r+\alpha-\beta_{k+1}}}\right\|_{p_{k}, \sigma_{l}^{\prime}}^{p_{k}-2}\left\|\frac{U^{(k+2 j-s)}}{\rho^{r+\alpha-\beta_{k+2 j-s}}}\right\|_{p_{k}, \sigma_{l}^{\prime}} \ll\|U\|_{W_{p_{k}, \alpha}^{r}\left(\sigma_{l}^{\prime}\right)}^{p_{k}-1}
$$

Если $p_{k}-s-2>0$, то, воспользовавшись неравенством Гёльдера с показателями

$$
\lambda_{1}=\frac{p_{k}}{q_{k}\left(p_{k}-2-s\right)}, \quad \lambda_{2}=\frac{p_{k}}{s q_{k}}, \quad \lambda_{3}=\frac{p_{k}}{q_{k}},
$$

получим

$$
Q \leqslant\left\|\frac{U^{(k)}}{\rho^{r+\alpha-\beta_{k}}}\right\|_{p_{k}, \sigma_{l}^{\prime}}^{p_{k}-2-s}\left\|\frac{U^{(k+1)}}{\rho^{r+\alpha-\beta_{k+1}}}\right\|_{p_{k}, \sigma_{l}^{\prime}}^{s}\left\|\frac{U^{(k+2 j-s)}}{\rho^{r+\alpha-\beta_{k+2 j-s}}}\right\|_{p_{k}, \sigma_{l}^{\prime}} \ll\|U\|_{W_{p_{k}, \alpha}^{r}\left(\sigma_{l}^{\prime}\right)^{p_{k}-1}} .
$$

В силу (35)-(38) получим

$$
\left\|\left|U^{(k)}\right|^{p_{k}-2-s}\left(U^{(k+1)}\right)^{s} U^{(k+2 j-s)}\right\|_{q_{k}, \sigma_{l}^{\prime}} \ll \rho^{\nu_{1}}\left(x_{0}\right)\|U\|_{W_{p_{k}, \alpha}^{r}\left(\sigma_{l}^{\prime}\right)}^{p_{k}-1} .
$$

Аналогично оцениваются слагаемые второй суммы из (33), а также слагаемые, составляющие первую и вторую сумму из равенства $(34)$, и $E_{0}$. Для них имеем такие же оценки, как $(39)$, только показатель $\nu_{1}$ надо заменить на $\nu_{2}, \nu_{3}, \nu_{4}, \nu_{5}$ соответственно:

$$
\begin{aligned}
& \nu_{2}=\left(p_{k}-2-s\right)\left(r+\alpha-\beta_{k}\right)+s\left(r+\alpha-\beta_{k+2}\right)+r+\alpha-\beta_{k+2(j-s)}, \\
& \nu_{3}=\left(p_{k}-2-s\right)\left(r+\alpha-\beta_{k}\right)+s\left(r+\alpha-\beta_{k+1}\right)+r+\alpha-\beta_{k+2 j-s-1}, \\
& \nu_{4}=\left(p_{k}-2-s\right)\left(r+\alpha-\beta_{k}\right)+s\left(r+\alpha-\beta_{k+2}\right)+r+\alpha-\beta_{k+2(j-s)-1}, \\
& \nu_{5}=\left(p_{k}-1\right)\left(r+\alpha-\beta_{k}\right) .
\end{aligned}
$$

Из определения $\beta_{k}$ (см. (4)) следует

$$
\beta_{k^{\prime}}-\beta_{k^{\prime \prime}} \geqslant k^{\prime}-k^{\prime \prime} \quad \forall k^{\prime}, k^{\prime \prime}, \quad k^{\prime} \leqslant k^{\prime \prime} \leqslant r .
$$

Поэтому

$$
-p_{k}\left(r+\alpha-\beta_{k}\right)-k+n+\nu_{j} \geqslant-r-\alpha+\beta_{k}-k, \quad j=1,2,3,4 .
$$

Теперь в силу $(31),(32),(39)$ для $J_{2}\left(\sigma_{l}^{\prime}, l\right)$ получим оценку

$$
\left|J_{2}\left(\sigma_{l}^{\prime}, l\right)\right| \ll \sum_{k \leqslant r / 2} \rho^{-r-\alpha+\beta_{k}-k}\left(x_{0}\right)\|U\|_{W_{p_{k}, \alpha}^{r}}^{p_{k}-1} X^{1 / p}\left(\sigma_{l}^{\prime}, l\right) .
$$


Нам осталось оценить интеграл $J_{3}\left(\sigma_{l}^{\prime}, l\right)$ :

$$
\begin{aligned}
\left|J_{3}\left(\sigma_{l}^{\prime}, l\right)\right| & =\left|\int_{\sigma_{l}^{\prime}} \frac{\Delta^{l} F}{h^{l}} A_{l} \eta_{l} d x\right|=\left|\int_{\sigma_{l}^{\prime}} F \frac{\Delta_{-h}^{l}}{h^{l}}\left(A_{l} \eta_{l}\right) d x\right| \\
& \leqslant\|F\|_{q, \sigma_{l}^{\prime}}\left\|\left(A_{l} \eta_{l}\right)^{(l)}\right\|_{p, \sigma_{l}^{\prime}} \ll \rho^{-r-\alpha+s_{0}}\left(x_{0}\right)\left\|\rho^{r+\alpha-s_{0}} F\right\|_{q, \sigma_{l}^{\prime}} X^{1 / p}\left(\sigma_{l}^{\prime}, l\right) .
\end{aligned}
$$

Из соотношений (18) при $\sigma^{\prime}=\sigma_{l}^{\prime}, \eta(x)=\eta_{l}(x),(30),(40),(41)$ следует

$$
\left\|\eta_{l}^{2 / q} \frac{\Delta^{l} \psi}{h^{l}}\right\|_{q, \sigma_{l}^{\prime}} \ll S\left(\sigma^{\prime}\right) .
$$

Отсюда, так как $\eta_{l}(x)=1$ на $\sigma_{l}$, имеем

$$
\left\|\frac{\Delta^{l} \psi}{h^{l}}\right\|_{q, \sigma_{l}} \ll S\left(\sigma^{\prime}\right), \quad l=m+1, \quad 1 \leqslant m<r .
$$

Неравенства (29) и, следовательно, неравенство (26) доказаны. Из (26) на основании неравенств $(8),(9)$ из $[2$, с. 116$]$ получим (см. $(24)$ для $\left.S\left(\sigma^{\prime}\right)\right)$

$$
\left\|\psi^{(r)}\right\|_{q, \sigma} \ll \rho^{-r-\alpha}\left(x_{0}\right)\left(\|U\|_{W_{p, \alpha}^{r}\left(\sigma^{\prime}\right)}^{p-1}+\sum_{k \leqslant r / 2}\|U\|_{W_{p_{k}, \alpha}^{r}\left(\sigma^{\prime}\right)}^{p_{k}-1}+\left\|\rho^{r+\alpha-s_{0}} F\right\|_{q, \sigma^{\prime}}\right) .
$$

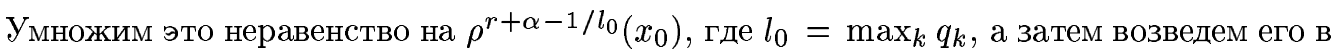
степень $q$ и проинтегрируем по $x_{0} \in \Delta=(a, b)$. Если полученньй результат возвести в степень $1 / q$ и применить к каждому члену теорему Труази, то получим неравенство (cм. [2, c. 103])

$$
\left\|\rho^{r+\alpha+\left(l_{0}-q\right) /\left(q l_{0}\right)} \psi^{(r)}\right\|_{q, \Delta} \ll\|U\|_{W_{p, \alpha}^{r}(\Delta)}^{p-1}+\sum_{k \leqslant r / 2}\|U\|_{W_{p_{k}, \alpha}^{r}(\Delta)}^{p_{k}-1}+\left\|\rho^{r+\alpha-s_{0}} F\right\|_{q, \Delta} .
$$

Отсюда, так как $2 \leqslant p_{k} \leqslant p$, следует неравенство (13). Аналогично, на основании (20) получим неравенство (12).

СЛЕДСТВИЕ. В условиях теоремы 3 имеют место неравенства

$$
\begin{gathered}
\left\|\rho^{\alpha} \psi\right\|_{q, \Delta}^{q} \ll\|\Phi\|_{W_{p, \alpha}^{r}(\Delta)}^{\gamma_{0}}+\left\|\rho^{r+\alpha-s_{0}} F\right\|_{q, \Delta}^{q}, \\
\left\|\rho^{r+\alpha+\left(l_{0}-q\right) /\left(q l_{0}\right)} \psi^{(r)}\right\|_{q, \Delta} \ll\left\|\rho^{r+\alpha-s_{0}} F\right\|_{q, \Delta}+\left(\|\Phi\|_{W_{p, \alpha}^{r}(\Delta)}^{\gamma_{0}}+\left\|\rho^{r+\alpha-s_{0}} F\right\|_{q, \Delta}^{q}\right)^{\lambda_{0} / p} .
\end{gathered}
$$

Неравенство (42) следует из (9), (12), а неравенство (43) - из (9), (13).

\section{СПИСОК ЦИТИРОВАННОЙ ЛИТЕРАТУРЫ}

[1] Никольский С. М. Приближение функций многих переменных и теоремы вложения. 2-е изд. М.: Наука, 1977.

[2] Лизоркин П. И., Никольский С. М. Коэрцитивное свойство эллиптических уравнений с вырождением. Вариационный метод // Тр. МИАН. 1981. Т. 157. С. 90-118.

[3] Салманов Ю. Д. Весовые классы функций с вырождением на многообразиях любых измерений // Докл. АН СССР. 1987. Т. 294. № 3. С. 539-542.

[4] Салманов Ю. Д. Следы функций из весовых классов на многообразиях и обратные теоремы вложения // Докл. АН СССР. 1991. Т. 319. № 4. С. 823-826. 